PROCEEDINGS OF THE

AMERICAN MATHEMATICAL SOCIETY

Volume 51, Number 1, August 1975

\title{
A NOTE ON PROPER MAPS
}

\author{
CHUNG-WU HO $^{1}$
}

ABSTRACT. The author establishes some necessary and sufficient conditions on a Hausdorff space $Y$ which force every open proper map into $Y$ to be surjective. Using this result, the author then shows that a local homeomorphism from a path connected space into a simply connected space is a global homeomorphism onto if and only if the map is proper.

A subset $A$ of a topological space $Y$ is called compactly closed if its intersection with each compact subset of $Y$ is closed in $Y$. A Hausdorff space is called a $k$-space if each of its compactly closed sets is closed. A map of a space $X$ into a space $Y$ is called open if it carries open subsets of $X$ onto open subsets of $Y$, and the map is called proper if the inverse image of each compact subset of $Y$ is a compact subset of $X$. It is frequently of interest to know that a given open proper map is surjective. Recently, Palais pointed out $[2$, p. 675] that any open proper map from a nonempty space into a connected $k$-space must be surjective, for it has been established that any proper map into a $k$-space is always closed [8]. If the map is also open, then the image of the map, being a set which is both open and closed in a connected space, must cover the whole space. In this note, we shall first establish some necessary and sufficient conditions on a space $Y$ which force every open proper map into $Y$ to be surjective. We shall then show that Palais' observation does not give a best possible criterion for an open proper map to be surjective. Using the results developed, we shall finally show that under mild restrictions, the properness of a map also provides a necessary and sufficient condition for a local homeomorphism to be a global homeomorphism.

Theorem 1. The following conditions on a Hausdorff space $Y$ are equivalent:

Received by the editors January 9, 1974 and, in revised form, June 7, 1974. AMS (MOS) subject classifications (1970). Primary 54C10, 54C05; Secondary $54 \mathrm{D} 50,57 \mathrm{D} 50$.

Key words and phrases. Compactly closed sets, $k$-space, proper map, local homeomorphism, covering projection, homotopy lifting property.

${ }^{1}$ The author would like to thank Professor William C. Bennewitz of Southern Illinois University at Edwardsville for many stimulating and helpful conversations.

Copyright (c) 1975. American Mathematical Society 
(a) The only open subsets of $Y$ which are compactly closed in $Y$ are the whole space and the empty set.

(b) Every open proper map from a nonempty space $X$ into $Y$ is surjective.

(c) Every injective open proper map from a nonempty space $X$ into $Y$ is a homeomorphism of $X$ onto $Y$.

Proof. To show (a) $\Rightarrow(b)$, consider any open proper map $f: X \rightarrow Y$ from some nonempty space $X$ into $Y$. Note that $f(X)$ is a nonempty open subset of $Y$ which is compactly closed in $Y$, for if $K$ is a compact subset of $Y$, $f(X) \cap K=f\left(f^{-1}(K)\right)$, which is compact, and hence, closed in $Y$.

(b) $\Rightarrow$ (c) is trivial.

(c) $\Rightarrow$ (a) follows from the observation that if $A$ is any nonempty open subset of $Y$ which is compactly closed in $Y$, then the inclusion map $j: A \rightarrow$ $Y$ must be an injective open proper map. Hence, $A=Y$.

Remark 1. Any connected $k$-space clearly satisfies condition (a) of the preceding theorem. In particular, any connected space which is locally compact or first countable (e.g. metrizable) satisfies our condition (a). Our result thus covers Palais' observation. The following lemma and remark show that our result is in fact stronger than Palais' observation.

Lemma 1. Condition (a) of Theorem 1 is satisfied by any topological space in which every two points lie in a compact connected subset.

Proof. Let $X$ be any such space and $A$ be any nonempty open subset of $X$ which is compactly closed in $X$. Suppose $A$ is not the whole space $X$. Then, we may find a compact connected subset $K$ of $X$ which intersects both $A$ and $X-A$. But then, $K \cap A$ would be both closed and open in $K$, and hence, identical with $K$. We get a contradiction.

Remark 2. By Lemma 1, any pathwise connected space certainly satisfies condition (a) of Theorem 1. However, it is well known that there are pathwise connected Hausdorff spaces which are not $k$-spaces. For instance see $[6$, p. 240 , Problem J(b)].

A map $f: X \rightarrow Y$ is called a local homeomorphism if each point of $X$ has an open neighbourhood which is carried by $f$ homeomorphically onto an open subset of $Y$. In the following, we shall use our results to establish a necessary and sufficient condition for a local homeomorphism between "nice" spaces to be a global homeomorphism. We first need the following two lemmas.

Lemma 2. Let $X$ and $Y$ be two Hausdorff spaces. Any surjective, proper, local homeomorphism $f: X \rightarrow Y$ is a covering projection if one of the fol. lowing conditions is satisfied: 
(a) $Y$ is locally compact.

(b) $\operatorname{card}\left(f^{-1}\left(y_{1}\right)\right)=\operatorname{card}\left(f^{-1}\left(y_{2}\right)\right)$ for any two points $y_{1}, y_{2}$ of $Y$.

Proof. We first consider an arbitrary surjective, proper, local homeomorphism $f: X \rightarrow Y$ without any further assumption. Consider any $y \in Y$. Since $f$ is a local homeomorphism, the set $f^{-1}(y)$ must be discrete. But $f$ is also proper, hence, $f^{-1}(y)$ must be finite. Let $f^{-1}(y)=\left\{x_{1}, x_{2}, \ldots, x_{n}\right\}$. By induction on $n$, we may easily find an open neighbourhood $U_{i}$ of $x_{i}$ such that the collection $\left\{U_{i}\right\}$ is pairwise disjoint. Replacing each $U_{i}$ by an open subset if necessary, we may further assume that $f$ carries each $U_{i}$ homeomorphically onto an open neighbourhood of $y$. Note that the set $V=\bigcap_{i=1}^{n} f\left(U_{i}\right)$ is an open neighbourhood of $y$, however, it may not be evenly covered by $f$ since the inverse image of $V$ might contain points which do not belong to the union of the $U_{i}$ 's. In fact, if no further assumption is imposed on $f$, one may construct a map $f: X \rightarrow Y$ which is not a covering projection.

Let us assume that condition (b) is satisfied by $f: X \rightarrow Y$. This condition guarantees that the inverse of the set $V=\bigcap_{i=1}^{n} f\left(U_{i}\right)$ contains no point outside the union of the $U_{i}^{\prime}$ 's. Hence, $V$ is a neighbourhood of $y$ which is evenly covered by $f$. Now, assume condition (a) is satisfied. We shall use the following construction given by Gordon [1, p. 758]. Let $K$ be a compact neighbourhood of $y$ and set

$$
V=\left(f\left(U_{1}\right) \cap \ldots \cap f\left(U_{n}\right)\right)-f\left[f^{-1}(K)-\left(b_{1} \cup \ldots \cup U_{n}\right)\right] .
$$

The inverse image of this set is in the union of the $U_{i}^{\prime}$ 's. Hence, $V$ is evenly covered by $f$. However, in this case, we need to show that $V$ is open, i.e., $f\left[f^{-1}(K)-\left(U_{1} \cup \cdots \cup U_{n}\right)\right]$ is closed. But this follows easily from the facts that $K$ is compact, $f$ is proper, and therefore, the set $f^{-1}(K)-$ $\left(U_{1} \cup \ldots \cup U_{n}\right)$ is compact.

Lemma 3. Let $X, Y$ be two Hausdorff spaces and let $Y$ be pathwise connected. Any surjective, proper, local homeomorphism $f: X \rightarrow Y$ must be a covering projection.

Proof. By the preceding lemma, we need only show that $\operatorname{card}\left(f^{-1}\left(y_{1}\right)\right)$ $=\operatorname{card}\left(f^{-1}\left(y_{2}\right)\right)$ for any two points $y_{1}$ and $y_{2}$ of $Y$. Consider any two such points $y_{1}$ and $y_{2}$. Let $\alpha: I \rightarrow Y$ be an arc in $Y$ from $y_{1}$ to $y_{2}$. Observe that the map $f \mid f^{-1}(\alpha(I)): f^{-1}(\alpha(I)) \rightarrow a(I)$ is a surjective, proper, local homeomorphism. Using Lemma 2 and the fact that $\alpha(I)$ is locally compact, we see that the restriction of $f$ to the set $f^{-1}(\alpha(I))$ is a covering projection. This clearly implies that $\operatorname{card}\left(f^{-1}\left(y_{1}\right)\right)=\operatorname{card}\left(f^{-1}\left(y_{2}\right)\right)$. 
Theorem 2. Let $X$ be pathwise connected and $Y$ be simply connected Hausdorff spaces. A local homeomorphism $f: X \rightarrow Y$ is a global homeomorphism of $X$ onto $Y$ if and only if the map $f$ is proper.

Proof. The only if part is clear. For the if part, we consider a proper, local homeomorphism $f: X \rightarrow Y$. Since any simply connected space is pathwise connected, Lemma 1 implies that $Y$ satisfies condition (a) of Theorem 1. Also observe that any local homeomorphism is an open map. Hence by the equivalence of (a) and (c) of Theorem 1, we need only show that $f$ is injective. But this can be established easily if one uses Lemma 3 and quotes the uniqueness of the universal covering space of $Y$. However, since the usual uniqueness theorem of the universal covering space requires the base space to be locally pathwise connected, we shall give an easy direct proof of the injectiveness of $f$.

Consider two points $x_{1}, x_{2}$, in $X$ such that $f\left(x_{1}\right)=f\left(x_{2}\right)=y$. Let $a$ be a path in $X$ connecting $x_{1}$ to $x_{2}$. Then $f \circ \alpha$ is a loop in $Y$ based at $y$. Since $Y$ is simply connected, we may find a homotopy $F: I \times I \rightarrow Y$ such that each $F_{t}$ is a loop at $y$ with $f_{0}=f \circ \alpha$ and $F_{1}=$ constant loop at $y$. Since a covering projection has the homotopy lifting property, we have a unique lifting $F^{\prime}$ of $F$ with $F_{0}^{\prime}=\alpha$. Then $\operatorname{Im}\left(F_{1}^{\prime}\right)$ must be a connected set containing both $x_{1}$ and $x_{2}$. But $\operatorname{Im}\left(F_{1}^{\prime}\right) \subset f^{-1}(y)$ and $f^{-1}(y)$ is a discrete set. Thus, $x_{1}=x_{2}$.

Remark 3. Our Theorem 2 also immediately implies the following generalized version of a classical theorem of Hadamard [3], [4], [5], which was recently rediscovered by Palais [7, pp. 128-129] and Gordon [1], [2]. Note that our version, stated below, is a somewhat improved version over that given by Gordon: ours may be applied to nonorientable manifolds and manifolds with or without boundaries.

Corollary. Let $M_{1}$ and $M_{2}$ be connected manifolds of class $C^{1}$ and suppose that $M_{2}$ is simply connected. Then a $C^{1}$ map $f$ from $M_{1}$ to $M_{2}$ is a diffeomorphism if and only if $f$ is proper and the Jacobian of $f$ never vanishes.

\section{BIBLIOGRAPHY}

1. W. B. Gordon, On the diffeomorphisms of euclidean space, Amer. Math. Monthly 79 (1972), 755-759. MR $46 \# 4548$.

2. - Addendum to "On the diffeomorphisms of euclidean space", Amer. Math. Monthly 80 (1973), 674-675. MR 47 \#7758.

3. J. Hadamard, Sur les transformations planes, C. R. Acad. Sci. Paris 142 (1906), 74. 
4. J. Hadamard, Sur les transformations ponctuelles, Bull. Soc. Math. France 34 (1906), 71-84; Oeuvres, pp. 349-363.

5. - Sur les correspondances ponctuelles, Oeuvres, pp. 383-384.

6. J. L. Kelley, General topology, Van Nostrand, Princeton, N. J., 1955. MR $16,1136$.

7. R. S. Palais, Natural operations on differential forms, Trans. Amer. Math. Soc. 92 (1959), 125-141. MR $22 \# 7140$.

8. - When proper maps are closed, Proc. Amer. Math. Soc. 24 (1970), 835836. MR $40 \# 8025$.

DEPARTMENT OF MATHEMATICS, SOUTHERN ILLINOIS UNIVERSITY, EDWARDSVILLE, ILLINOIS 62025 